\title{
Universal envelope equation and emittance evolution of high-brightness beam in linac
}

\author{
Chun-xi Wang* \\ Argonne National Laboratory, 9700 South Cass Avenue, Illinois 60439, USA
}

(Received 8 August 2008; published 23 April 2009)

\begin{abstract}
We report a universal beam envelope equation that governs the transverse linear dynamics of highintensity and high-brightness relativistic beams under constant acceleration in axisymmetric linear accelerators. This dimensionless and almost parameter-free nonlinear equation is useful for understanding scaling properties and for investigating nonlinear behaviors that are beyond analytical analysis. Particularly, we explore emittance compensation in high-brightness beams evolving from the spacecharge regime to the thermal-emittance regime, a transition that commonly occurs during acceleration but is not well studied. A new formula is given for correctly computing the rms bunch emittance from slice envelopes, which is different from the commonly used quadratic sum of the thermal emittance and the rms emittance in the envelope phase space.
\end{abstract}

DOI: 10.1103/PhysRevSTAB.12.044201

PACS numbers: 29.27.Bd, 29.20.Ej, 29.25.Bx, 41.75.-i

\section{INTRODUCTION}

This paper deals with transverse linear beam dynamics during acceleration of space-charge-dominated highbrightness relativistic beams in axisymmetric accelerators. Our motivation comes from photoinjectors, but the results may also be useful to certain high-intensity proton or ion accelerators. Photoinjectors are the premier sources for high-brightness and high-intensity electron beams that drive many exciting applications such as $\mathrm{x}$-ray freeelectron lasers, the next-generation synchrotron-radiation sources based on energy-recovery linacs, and potentially the International Linear Collider. The performance and cost of these large, sophisticated devices rely critically on the quality of electron beams, especially the electron density in phase space. Over the past two decades, extensive research and development have been devoted to understanding and improving beam quality with lower emittance and higher current. A major difficulty is due to the repulsive Coulomb forces that tend to blow up high-density electron bunches at low energy. Because of cancellation between the Coulomb force and the self-magnetic force of a bunch moving at high velocity, the space-charge forces are inversely proportional to the square of the beam energy and are less harmful at high energy. Therefore, a primary goal in photoinjectors is to preserve the low thermal emittance that the electrons are born with, while accelerating the electrons from the space-charge regime at low energy to the thermal-emittance regime at high energy. To limit the space-charge disruption, the high electric field from a radio-frequency (rf) cavity is used around a cathode to accelerate electrons from rest to relativistic energy as quickly as possible. Even so, in the early days, large

\footnotetext{
*wangcx@aps.anl.gov;

URL: http://www.aps.anl.gov/ wangcx
}

correlated transverse emittance growth was observed, mostly due to linear space-charge forces.

A key advance in rf photoinjector development is the recovery of the initial emittance with proper focusing, a technique known as emittance compensation [1,2]. This critical process is governed by the nonlinear envelope equation addressed shortly. The first beam-envelope theory of emittance compensation is based on perturbative treatment of space-charge-dominated beam evolution in constant focusing channels nearby a special quasiequilibrium solution, known as the invariant envelope $[3,4]$. For spacecharge-dominated beams, the invariant envelope is analogous to Brillouin flow for nonaccelerating beams, where focusing and defocusing forces are balanced to produce a stable beam. This theory successfully revealed a matching condition for a space-charge-dominated beam at the entrance of a linac. However, it is inadequate for emittance compensation inside an injector where the beam is far from equilibrium and there is no well-defined invariant envelope. This problem was addressed recently, and additional criteria for compensating the detrimental slice-dependent space-charge and chromatic forces were found [5]. Current understanding of emittance compensation in linacs is still limited to the space-charge-dominated invariant-envelope theory. However, as a beam is accelerated out of the spacecharge regime, this model inevitably fails because the emittance term must be considered. Furthermore, the invariant envelope becomes unstable due to insufficient focusing, and nonlinearity has to be taken into account. Unfortunately, the intrinsic nonlinearity makes it difficult to develop a more general theory until now, and general dynamics across the transition (from space-charge regime to emittance regime) is not well understood. In this paper we present a dimensionless and almost parameter-free universal beam envelope equation for a relativistic beam under constant acceleration. It provides an opportunity to numerically explore emittance compensation beyond ap- 
proximate theory and obtain understanding as general as analytical theories can provide.

When using the slice envelopes to compute rms bunch emittance, a common practice is to use the rms sliceenvelope emittance in the envelope phase space, i.e., $\sqrt{\left\langle\sigma^{2}\right\rangle\left\langle\sigma^{\prime 2}\right\rangle-\left\langle\sigma \sigma^{\prime}\right\rangle^{2}}$, or its quadratic sum with the thermal emittance. We pointed out that the correct expression should be $\sqrt{\left\langle\sigma^{2}\right\rangle\left\langle\sigma^{\prime 2}\right\rangle-\left\langle\sigma \sigma^{\prime}\right\rangle^{2}+\epsilon_{\mathrm{th}}^{2}\left\langle\sigma^{2}\right\rangle\left\langle 1 / \sigma^{2}\right\rangle}$, where $\sigma$ and $\epsilon_{\mathrm{th}}$ are the slice envelope and thermal emittance, respectively. The correct account of thermal-emittance effect becomes important as a beam comes out of the space-charge-dominated regime in a linac.

\section{UNIVERSAL BEAM ENVELOPE EQUATION}

Photoinjectors are usually axisymmetric. Evolution of a beam slice under linear forces (from external electromagnetic fields and average internal space-charge field) is governed by the reduced beam envelope equation $[4,6]$

$$
\hat{\sigma}^{\prime \prime}+\frac{\kappa}{\beta^{2} \gamma^{2}} \hat{\sigma}-\frac{\kappa_{s}}{\beta^{2} \gamma^{2}} \frac{1}{\hat{\sigma}}-\frac{\epsilon_{n}^{2}}{\hat{\sigma}^{3}}=0,
$$

where the reduced envelope $\hat{\sigma}$ is derived from the rms beam radius $\sigma$ by $\hat{\sigma}=\sqrt{\beta \gamma} \sigma ; \beta$ and $\gamma$ are the velocity divided by $c$ and relativistic energy factor of a reference particle, respectively; $\kappa$ is external focusing strength; $\kappa_{s}$ is beam perveance (generalized perveance without $\beta$ and $\gamma$ ); and $\epsilon_{n}$ is normalized slice emittance. We consider systems with constant $\epsilon_{n}$ and $\kappa_{s}$. Constant slice emittance requires linearity in the underlying single-particle dynamics, with negligible nonlinear space-charge force, in particular. The projected emittance of a bunch slice ensemble can still vary dramatically and needs to be controlled by emittance compensation. When a beam is matched into a linac, $\kappa_{s}$ often becomes constant and the same for all slices such as in the SPARC photoinjector $[5,7,8]$. The envelope equation has two defocusing terms, with the space-charge (emittance) term dominating at low (high) energy. The ratio of the emittance to the space-charge term

$$
\rho \equiv\left(\frac{\beta \gamma \epsilon_{n}}{\sqrt{\kappa_{s}} \hat{\sigma}}\right)^{2}
$$

is an important parameter that grows with acceleration from $\rho \ll 1$ (space-charge-dominated) to $\rho \gg 1$ (emittance dominated). The transition energy, defined by $\rho=$ 1 and not to be confused with the well-known transition associated with zero slippage, reads

$$
\left.\beta \gamma\right|_{\rho=1}=\frac{\sqrt{\kappa_{s}} \hat{\sigma}}{\epsilon_{n}}=\frac{\kappa_{s}}{\epsilon_{n} \sqrt{\kappa}},
$$

at which point the beam envelope changes from spacecharge-dominated to emittance dominated. A major result of understanding space-charge-dominated high-brightness beams is finding the invariant-envelope solution

$$
\hat{\sigma}_{\text {inv }}=\sqrt{\kappa_{s} / \kappa}
$$

and the emittance compensation of a beam close to the invariant envelope [3]. The invariant envelope is the obvious equilibrium solution of Eq. (1) with constant $\kappa$ and $\kappa_{s}$, and $\epsilon_{n}=0$. The envelope equation can be generated from a nonautonomous Hamiltonian with a timedependent nonlinear potential well, at whose bottom the invariant envelope sits [4]. However, such understanding is insufficient when both space-charge and emittance terms have to be considered.

Using the invariant envelope and the space-charge parameter $\rho$, the envelope equation can be written as

$$
\hat{\sigma}^{\prime \prime}+\frac{\bar{\kappa}}{\beta^{2} \gamma^{2}} \hat{\sigma}\left[1+\frac{\Delta \kappa}{\bar{\kappa}}-\left(\frac{\hat{\sigma}_{\text {inv }}}{\hat{\sigma}}\right)^{2}-\rho\left(\frac{\hat{\sigma}_{\text {inv }}}{\hat{\sigma}}\right)^{4}\right]=0,
$$

where we have extracted a constant part $\bar{\kappa}$ from the external focusing $\kappa$ and let $\Delta \kappa=\kappa-\bar{\kappa}$. The focusing constant $\bar{\kappa}$ is used for the invariant envelope in Eq. (4). Since beam energy and $\rho$ increase monotonically for accelerating beams, we can use beam energy (scaled by the transition energy)

$$
\bar{\rho} \equiv \frac{\sqrt{\rho}}{\beta}=\frac{\epsilon_{n}}{\sqrt{\kappa_{s}} \hat{\sigma}_{\text {inv }}} \gamma(s)
$$

as the independent variable instead of position $s$. Using the scaled dimensionless envelope

$$
w \equiv \frac{\hat{\sigma}}{\hat{\sigma}_{\text {inv }}}
$$

as the dependent variable instead of $\hat{\sigma}$, Eq. (1) reduces to

$\bar{\rho}^{2} \frac{d^{2} w}{d \bar{\rho}^{2}}+\frac{\gamma \gamma^{\prime \prime}}{\gamma^{\prime 2}} \bar{\rho} \frac{d w}{d \bar{\rho}}+\frac{\bar{\kappa}}{\beta^{2} \gamma^{\prime 2}}\left(w-\frac{1}{w}+\frac{\Delta \kappa}{\bar{\kappa}} w-\frac{\beta^{2} \bar{\rho}^{2}}{w^{3}}\right)=0$.

In deriving this equation we have used

$$
\hat{\sigma}^{\prime \prime}=\bar{\rho}^{\prime 2} \frac{d^{2} \hat{\sigma}}{d \bar{\rho}^{2}}+\bar{\rho}^{\prime \prime} \frac{d \hat{\sigma}}{d \bar{\rho}}, \quad \bar{\rho}^{\prime}=\frac{\gamma^{\prime}}{\gamma} \bar{\rho}, \quad \bar{\rho}^{\prime \prime}=\frac{\gamma^{\prime \prime}}{\gamma} \bar{\rho} .
$$

Hereafter, primes on $\hat{\sigma}, \gamma$, and $\bar{\rho}$ represent differentiation with respect to position $s$, but primes on $w$ represent differentiation with respect to $\bar{\rho}$. Equation (8) can be derived from the Hamiltonian

$$
\begin{aligned}
H= & \frac{\sqrt{\kappa_{s}}}{2 \gamma^{\prime} \epsilon_{n}}\left[p_{w}^{2}+\left(\frac{\epsilon_{n}}{\hat{\sigma}_{\text {inv }}}\right)^{2} \frac{1}{\beta^{2} \bar{\rho}^{2}}\right. \\
& \left.\times\left(w^{2}-\ln w^{2}+\frac{\Delta \kappa}{\bar{\kappa}} w^{2}+\frac{\beta^{2} \bar{\rho}^{2}}{w^{2}}\right)\right]
\end{aligned}
$$

with a time-dependent nonlinear potential well.

In linacs, to a good approximation (for beams from highbrightness photoinjectors where the beam becomes relativ- 
istic although it is still space-charge dominated; more discussion later), we have

$$
\beta \simeq 1, \quad \gamma^{\prime \prime} \simeq 0
$$

The focusing from $\mathrm{rf}$ accelerating field of amplitude $E_{0}$ and solenoid of amplitude $B_{s}$ is given by $[3,4]$

$$
\kappa=\left\langle\gamma^{\prime}\right\rangle^{2}\left(\Omega^{2}+\frac{1}{4}\right), \quad \Omega^{2} \equiv \frac{1}{\sin ^{2} \varphi_{\mathrm{r}}}\left[\frac{\eta}{8}+\left(\frac{B_{s} c}{E_{0}}\right)^{2}\right],
$$

where $\varphi_{\mathrm{r}}$ is the $\mathrm{rf}$ phase of the reference particle, $\eta$ is determined by the space harmonics of the accelerating structure, and $c$ is the speed of light. Insert these into Eq. (8) and, to our surprise, Eq. (1) reduces to a universal envelope equation

$$
\bar{\rho}^{2} \frac{d^{2} w}{d \bar{\rho}^{2}}+\left(\bar{\Omega}^{2}+\frac{1}{4}\right)\left(w-\frac{1}{w}+\frac{\Omega^{2}-\bar{\Omega}^{2}}{\bar{\Omega}^{2}+1 / 4} w-\frac{\bar{\rho}^{2}}{w^{3}}\right)=0,
$$

where all the quantities are dimensionless, and $\bar{\Omega}$ is a focusing constant associated with $\bar{\kappa}$ via Eq. (12) and the invariant envelope (e.g., initial value of $\Omega$ at the linac entrance). This equation is remarkable because of its universal form, independent of beam parameters such as emittance, perveance, energy, and accelerating gradient. In fact, there is no free parameter at all for a traveling-wave linac with $\Omega=0$. Despite its universal and parameter-free form, the universal envelope equation is almost as good as the original envelope equation. This is significant because it reduces complex problems to a unique parameter-free equation that can be studied numerically without losing generality. Furthermore, it provides a foundation to understand various scaling properties in such systems. Note that a different universal equation has been used in [3], but it is limited to the space-charge regime. Existence of a general universal envelope equation is nontrivial and useful. We should also mention that different scaling methods have long been used to reduce free parameters in the envelope equation for intense beams in constant-focusing channels without acceleration (e.g., [9]).

Because of the intrinsic nonlinearities in the envelope equation, it is notoriously hard to draw analytical conclusions. However, numerical solutions are straightforward, from which general conclusions on beam evolution can be obtained, thanks to the universal form of the envelope equation. In the following, we investigate beam evolution from the space-charge regime to the emittance regime, a transition that commonly occurs in high-brightness beams but for which little is known about its general dynamics (except numerous design simulations).

\section{UNIVERSAL BEAM ENVELOPE UNDER UNIFORM FOCUSING}

In the case of constant focusing, the universal envelope equation has only one constant parameter $\bar{\Omega}$, and the $\Omega^{2}-$ $\bar{\Omega}^{2}$ term in Eq. (13) vanishes. Current emittancecompensation theory in a linac is based on linear perturbation around $w=1$, assuming $\bar{\rho} \ll 1$ due to space-charge domination. As $\bar{\rho}$ inevitably becomes large, $w=1$ is no longer a solution, and the perturbation diverges with beam energy [3,4] (see the $\bar{\rho}$ dependence of the $R$ matrix in the Appendix). To see what actually happens, we used the universal envelope equation to numerically compute the special solution $W_{\Omega}(\bar{\rho})$ satisfying $W_{\Omega}(0)=1$ and $W_{\Omega}^{\prime}(0)=0$, the initial conditions for the invariant envelope. Figure 1 plots $W_{\Omega}$ and its derivative for $\Omega=0,1,2$, $3,4,5$ from $\bar{\rho}=0$ to 1 and 20 , respectively. It shows that the invariant envelope does not change much when $\bar{\rho} \ll 1$, but becomes unstable and expands freely as it goes through the transition $\bar{\rho} \simeq 1$.

The nonlinear potential in the Hamiltonian of Eq. (10) has a minimum given by

$$
w=\sqrt{1 / 2+\sqrt{1 / 4+\bar{\rho}^{2}}}
$$

In the space-charge regime, this potential minimum provides an equilibrium solution of the envelope equation, i.e., the invariant-envelope solution $w=1$. Figure 1 shows that, with strong focusing ( $\Omega \gtrsim 3$ ), the invariant envelope still sits at the potential minimum even outside the spacecharge regime, which has been proposed to describe the transition $[10,11]$. However, this may not be suitable for weak focusing with smaller $\Omega$, especially when there is no magnetic focusing. The qualitative behavior of $w^{\prime}$ is easy to understand. From $w^{\prime \prime}=\bar{\rho}^{-2}\left(\Omega^{2}+1 / 4\right)\left(1 / w+\bar{\rho}^{2} / w^{3}-\right.$ $w)$ we see that Eq. (14) gives the turning point, where $w^{\prime \prime}=$ 0 and $w^{\prime}$ reaches maximum and then decreases.

Using the universal envelope $W_{\Omega}$, just like a mathematical special function, a general beam envelope initially matched to the invariant envelope can be expressed as

$$
\sigma=\sqrt{\frac{\kappa_{s}}{\gamma \bar{\kappa}}} W_{\Omega}\left(\frac{\gamma \epsilon_{n} \sqrt{\bar{\kappa}}}{\kappa_{s}}\right) .
$$

Note that the accelerating gradient has no role in it. The $1 / \sqrt{\gamma}$ in front of $W_{\Omega}$ reflects the well-known adiabatic damping with energy, which is stopped after transition by the $\gamma$ dependence of $W_{\Omega}$.

Besides providing a better understanding of the basic dynamics, such a solution offers a new way to compute the often-needed linear transfer matrix across an accelerating structure for intense beams matched to the invariant envelope, taking linear space-charge forces into account. 


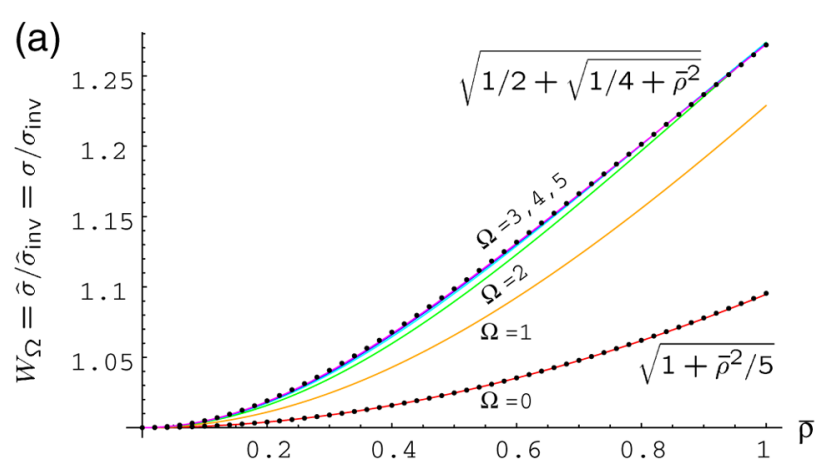

\section{EMITTANCE EVOLUTION}

In addition to the invariant-envelope evolution, it is important to know the evolution of the emittance. Using the slice envelopes, the rms bunch emittance $\epsilon_{\text {rms }}$ can be computed as

$$
\begin{aligned}
\epsilon_{\mathrm{rms}}^{2} & =\left|\left[\begin{array}{cc}
\left\langle x^{2}\right\rangle_{\text {bunch }} & \left\langle x x^{\prime}\right\rangle_{\text {bunch }} \\
\left\langle x x^{\prime}\right\rangle_{\text {bunch }} & \left\langle x^{\prime 2}\right\rangle_{\text {bunch }}
\end{array}\right]\right| \\
& =\left|\left\langle\left\langle\left[x, x^{\prime}\right]^{T}\left[x, x^{\prime}\right]\right\rangle_{s}\right\rangle_{\text {slices }}\right| \\
& =\left|\left\langle\left[\begin{array}{cc}
\sigma^{2} & \sigma \sigma^{\prime} \\
\sigma \sigma^{\prime} & \sigma^{\prime 2}+\epsilon_{n}^{2} / \sigma^{2}
\end{array}\right]\right\rangle_{\text {slices }}\right| \\
& =\left\langle\sigma^{2}\right\rangle\left\langle\sigma^{\prime 2}\right\rangle-\left\langle\sigma \sigma^{\prime}\right\rangle^{2}+\left\langle\sigma^{2}\right\rangle\left\langle\epsilon_{n}^{2} / \sigma^{2}\right\rangle,
\end{aligned}
$$

where $\langle\cdots\rangle_{\text {bunch }},\langle\cdots\rangle_{s}$, and $\langle\cdots\rangle_{\text {slices }}$ indicate ensemble average over the entire bunch, an individual slice, and all slices, respectively. $\epsilon_{n}^{2} \equiv\left\langle x^{2}\right\rangle_{s}\left\langle x^{\prime 2}\right\rangle_{s}-\left\langle x x^{\prime}\right\rangle_{s}^{2}$ is the rms emittance of individual slices. Slice envelope $\sigma^{2} \equiv\left\langle x^{2}\right\rangle_{s}$, whose derivative yields $\left\langle x x^{\prime}\right\rangle_{s}=\sigma \sigma^{\prime}$, and thus $\left\langle x^{\prime 2}\right\rangle=$ $\sigma^{\prime 2}+\epsilon_{n}^{2} / \sigma^{2}$, which has been used in the above emittance expression. The second derivative of beam envelope yields $\sigma \sigma^{\prime \prime}+\sigma^{\prime 2}=\left\langle x x^{\prime \prime}\right\rangle_{s}+\left\langle x^{\prime 2}\right\rangle_{s}$. In general, such beammoment equations cannot be closed. But for linear forces with $x^{\prime \prime}=-K(s) x$, this equation gives the slice-envelope equation $\sigma^{\prime \prime}+K \sigma-\epsilon_{n}^{2} / \sigma^{3}=0$. Furthermore, $\epsilon_{n}$ becomes constant and usually the same for all slices. We review these details because, to our knowledge, all sliceenvelope-based emittance computations have been using the envelope-phase-space emittance $\sqrt{\left\langle\sigma^{2}\right\rangle\left\langle\sigma^{\prime 2}\right\rangle-\left\langle\sigma \sigma^{\prime}\right\rangle^{2}}$, which is Eq. (16) without the thermal-emittance term $\epsilon_{n}^{2}\left\langle\sigma^{2}\right\rangle\left\langle 1 / \sigma^{2}\right\rangle$ in the square root (sometimes, thermal emittance is accounted for by a simple-minded quadratic sum without the factor $\left.\left\langle\sigma^{2}\right\rangle\left\langle 1 / \sigma^{2}\right\rangle\right)$. However, though appealing, the envelope-phase-space emittance may not be adequate for computing particle-phase-space emittance, especially when thermal emittance becomes important. It should be clear that the rms bunch emittance in Eq. (16) has not taken into account nonlinear effects such as filamentation of mismatched beams. Thus, it may not represent the final emittance of a thermalized beam in a long linac.

Inserting $\hat{\sigma}=\hat{\sigma}_{\text {inv }} w=\sqrt{\kappa_{s} / \bar{\kappa}} w$ and $\hat{\sigma}^{\prime}=\bar{\rho}^{\prime} \hat{\sigma}_{\text {inv }} w^{\prime}=$ $\left(\gamma^{\prime} \epsilon_{n} / \sqrt{\kappa_{s}}\right) w^{\prime}$ into Eq. (16), the rms emittance $\epsilon_{\sigma}$ in the $\hat{\sigma}$ coordinates reduces to

$$
\epsilon_{\sigma}=\frac{\epsilon_{n}}{\sqrt{\bar{\kappa}} / \gamma^{\prime}} \epsilon_{w}=\frac{\epsilon_{n}}{\sqrt{\bar{\Omega}^{2}+1 / 4}} \epsilon_{w},
$$

FIG. 1. (Color) Universal beam envelope $W_{\Omega}(\bar{\rho})$, which represents evolution of the invariant envelope under constant focusing and acceleration. Plots (a) and (b) are $W_{\Omega}$ in the space-charge regime ( $\bar{\rho}$ from 0 to 1$)$ and up to the emittance regime ( $\bar{\rho}$ from 0 to 20), respectively. Plots (c) and (d) are the corresponding derivatives $W_{\Omega}^{\prime}$. The curves are for different values of focusing parameter $\Omega$, as labeled. The dots are approximate solutions with the given expressions. Clearly, the invariant envelope is unstable and transits into free expansion. For strong focusing with large $\Omega$, the approximate solution given by the potential minimum appears good all the way through the transition.

where $\epsilon_{w}$ is the emittance in the $w$ coordinates given by

$\epsilon_{w}=\sqrt{\left\langle\sigma_{w}^{2}\right\rangle\left\langle\sigma_{w}^{\prime}{ }^{2}\right\rangle-\left\langle\sigma_{w} \sigma_{w}^{\prime}\right\rangle^{2}+\left(\bar{\Omega}^{2}+1 / 4\right)\left\langle\sigma_{w}^{2}\right\rangle\left\langle 1 / \sigma_{w}^{2}\right\rangle}$.

Note that $\sqrt{\bar{\Omega}^{2}+1 / 4}$ plays the role of thermal emittance in the $w$ space. 
A conceptual benefit of using the naturally scaled variables and the beam-independent universal equation is the possibility to address optics issues independent of beam properties. In particular, since $\left\langle\sigma_{w}^{2}\right\rangle\left\langle\sigma_{w}^{\prime}{ }^{2}\right\rangle-\left\langle\sigma_{w} \sigma_{w}^{\prime}\right\rangle^{2} \geq 0$ and $\left\langle\sigma_{w}^{2}\right\rangle\left\langle 1 / \sigma_{w}^{2}\right\rangle \geq 1$ according to Schwarz and Chebyshev inequalities, $\epsilon_{w} \geq \sqrt{\bar{\Omega}^{2}+1 / 4}$. Clearly it is desirable to have the envelope-phase-space emittance

$$
\sqrt{\left\langle\sigma_{w}^{2}\right\rangle\left\langle\sigma_{w}^{\prime}{ }^{2}\right\rangle-\left\langle\sigma_{w} \sigma_{w}^{\prime}\right\rangle^{2}}<\sqrt{\bar{\Omega}^{2}+1 / 4}
$$

and

$$
\left\langle\sigma_{w}^{2}\right\rangle\left\langle 1 / \sigma_{w}^{2}\right\rangle \simeq 1
$$

These simple expressions help to set a clear goal for the envelope-phase-space distribution at the high-energy end in the emittance-dominated regime, while the universal envelope equation can map it back to the emittancedominated regime at the entrance of a linac, regardless of the various parameters of a particular system.

To investigate the evolution of the emittance across the transition, we compute $\epsilon_{w}$ using Eq. (18) and our parameter-free universal envelope equation with $\Omega=0$. Starting from a slice ensemble randomly distributed on an ellipse in the $w-w^{\prime}$ space around the invariant envelope at $\bar{\rho}=0.1$ (see the first inset in Fig. 2), we numerically track each slice and plot the emittance evolution in Fig. 2 up to $\bar{\rho}=10$. The rms emittance is computed using, respectively, the exact universal equation (red and black), the space-charge-dominated theory that assumes $\epsilon_{n}=0$ and linear perturbation around $w=1$ (cyan), and linear perturbation around $W_{\Omega}$ (blue). The red curve includes the thermal emittance while the other three do not. The evo-

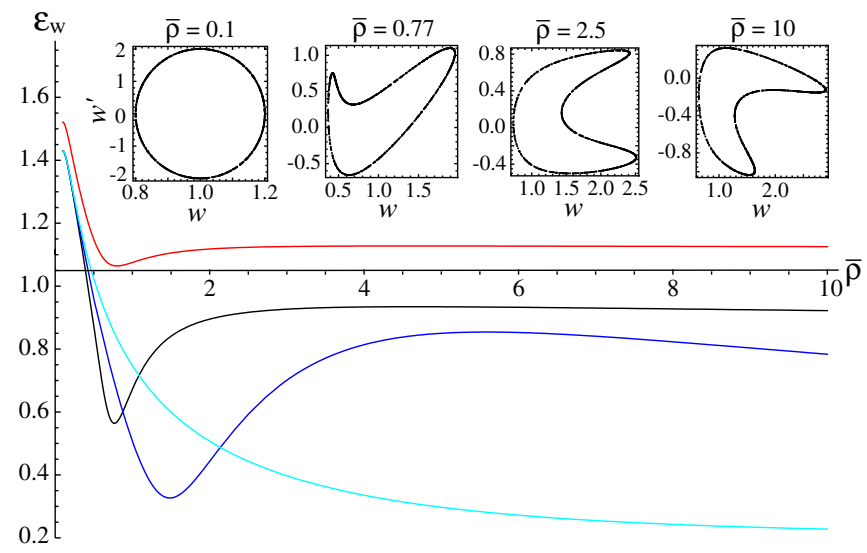

FIG. 2. (Color) Emittance compensation in linacs with constant focusing. The red curve is the rms bunch emittance computed with the universal equation and Eq. (18). The other three curves are rms envelope-phase-space emittance from, respectively, the exact universal equation (black), the space-charge-dominated theory (cyan), and linear perturbation around $W_{\Omega}$ (blue). Insets are phase-space distributions at the beginning and the end, as well as at locations with the minimum and maximum emittances. lution of an envelope-phase-space ellipse is used to demonstrate the nonlinear effects, since linear dynamics will simply map an ellipse to other ellipses. The emittance decreases at the beginning mostly due to adiabatic damping, then increases as nonlinearities pick up. Space-chargedominated theory clearly fails across the transition. The effect of thermal emittance is significant across transition into the emittance-dominated regime.

To examine the relevance and accuracy of our theory in practice, we apply it to the state-of-art SPARC photoinjector $[7,8]$, which has been analyzed in [5] with notations consistent with this paper. Its booster linac consists of two accelerating cavities separated by a short drift. A focusing solenoid is superimposed on the first cavity. Detailed focusing profile as well as perveance and energy of the center slice are given in Fig. 1 of [5]. For our purpose here, from a HOMDYN [12] simulation of this injector, we extracted the phase-space information of all slices at the booster entrance and converted it into $w-w^{\prime}$ space, then numerically tracked all slices using the universal envelope equation, Eq. (13), with the solenoid focusing treated as $\Delta \kappa$ and the drift ignored. The resulting emittance is plotted in Fig. 3 together with the HOMDYN simulation. The beam is accelerated from about $\bar{\rho} \sim 0.05$ at $6 \mathrm{MeV}$ to $\bar{\rho} \sim 1.3$ at $156 \mathrm{MeV}$, barely across the transition. The agreement is excellent except for a small shift in the second cavity due to the drift space. To see beam behavior further into the emittance regime, Fig. 3 also shows the emittance up to $\bar{\rho}=4$ under the same acceleration. To see the effect of thermal emittance on rms bunch emittance, we showed, respectively, the envelope-phase-space emittance itself (blue and cyan), its quadratic sum with the thermal emittance (magenta), and rms bunch emittance computed with Eq. (18).

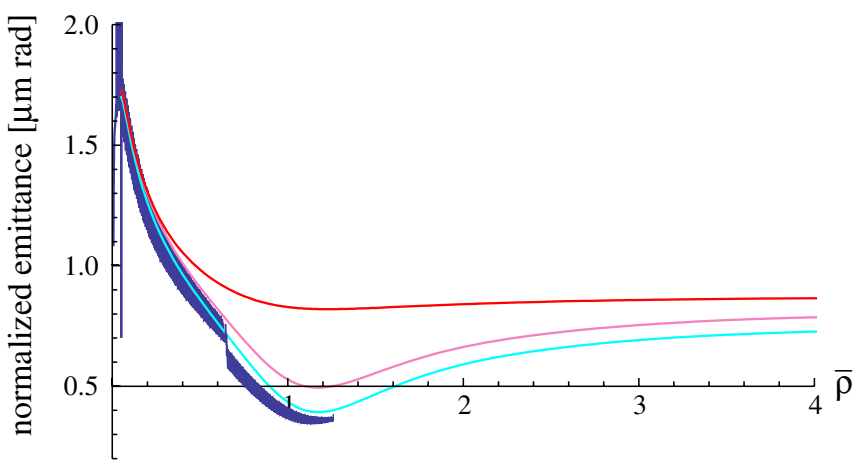

FIG. 3. (Color) Comparison of emittance in the booster linac of the SPARC photoinjector computed using HOMDYN simulation (blue) and the universal envelope equation (cyan) excluding the thermal emittance. The small difference in the second cavity is due to the drift space between the two cavities in the booster. The emittance beyond the booster is also shown assuming the same accelerating structures. Thermal emittance is included in the red curve using Eq. (18) and in the magenta curve using a simple quadratic sum. 


\section{DISCUSSIONS}

In deriving the universal envelope equation, we have assumed a space-charge-dominated beam that is relativistic and under constant acceleration in Eq. (11). This is true for present-day high-brightness photoinjectors because of the extremely small emittance and high bunch charge. In our example-the SPARC injector shown in Fig. 3-the beam is space-charge-dominated beyond $100 \mathrm{MeV}$. However, it may be desirable to remove the $\beta=1$ condition for other applications, especially for proton and ion linacs. To explore this possibility, we note that, if using $\bar{\rho} \equiv \sqrt{\rho}$ instead of Eq. (6), Eq. (8) changes to

$\bar{\rho}^{2} \frac{d^{2} w}{d \bar{\rho}^{2}}+F(\gamma) \bar{\rho} \frac{d w}{d \bar{\rho}}+\frac{\beta^{2} \bar{\kappa}}{\gamma^{\prime 2}}\left(w-\frac{1}{w}+\frac{\Delta \kappa}{\bar{\kappa}} w-\frac{\bar{\rho}^{2}}{w^{3}}\right)=0$,

where $F(\gamma)=\frac{\beta^{2} \gamma \gamma^{\prime \prime}}{\gamma^{\prime 2}}-\frac{1}{\gamma^{2}}=-\frac{1}{\gamma^{2}}$ under constant acceleration. To estimate the effect of $F(\gamma)$, we consider the Wronskian determinant of linear perturbations that characterizes the effect of $F$ on mapping a small phase-space area, which is given by the factor [13]

$$
e^{-\int_{\bar{\rho}_{0}}^{\bar{\rho}} F(\gamma) / \bar{\rho} d \bar{\rho}}=e^{\int_{\gamma_{0}}^{\gamma} 1 / \gamma^{3} d \gamma}=e^{1 / 2 \gamma_{0}^{2}-1 / 2 \gamma^{2}} \leq e^{1 / 2 \gamma_{0}^{2}} .
$$

This suggests that we may be able to ignore the second term in the above equation when beam energy $\gamma>2$ with $10 \%$ accuracy.

\section{ACKNOWLEDGMENTS}

We give special thanks to M. Ferrario for providing the HOMDYN simulation of the SPARC photoinjector used in Fig. 3. This work was supported by U.S. Department of Energy, Office of Science, Office of Basic Energy Sciences, under Contract No. DE-AC02-06CH11357.

\section{APPENDIX A: LINEAR PERTURBATIONS}

The material in this Appendix is not necessary for understanding this paper, but can be interesting for understanding the evolution of small perturbation around the invariant envelope, especially the emittance effect on the transfer matrix discussed in $[3,4,14]$. In addition to a special solution $\bar{w}$, such as the invariant-envelope solution, we are also interested in nearby envelope solutions $w=\bar{w}+\delta w$. Inserting this into the universal envelope equation and linearizing the equation in terms of $\delta w$ yields

$\bar{\rho}^{2} \frac{d^{2} \delta w}{d \bar{\rho}^{2}}+\left(\bar{\Omega}^{2}+\frac{1}{4}\right)\left(1+\frac{1}{\bar{w}^{2}}+\frac{\Omega^{2}-\bar{\Omega}^{2}}{\bar{\Omega}^{2}+1 / 4}+\frac{3 \bar{\rho}^{2}}{\bar{w}^{4}}\right) \delta w=0$.

We are interested in solutions nearby the envelope starting from the invariant envelope $\bar{w}=1$. For a constant- focusing channel, $\bar{w} \simeq 1$ holds within a few percent up to $\bar{\rho}=1$ for $\Omega=0$ [worse for larger $\Omega$, as seen from $W_{\Omega}$ in Fig. 1(a)], and the $\Omega^{2}-\bar{\Omega}^{2}$ term is zero. Thus, we have the linear equation for small perturbations as

$$
\bar{\rho}^{2} \frac{d^{2} \delta w}{d \bar{\rho}^{2}}+\left(\bar{\Omega}^{2}+\frac{1}{4}\right)\left(2+\Lambda \bar{\rho}^{2}\right) \delta w=0,
$$

where $\Lambda=3$ for constant focusing. Setting $\Lambda=0$ will ignore the emittance effects and reduce to the equation studied in $[3,4,14]$.

This equation can be transformed into the wellknown Bessel equation $d^{2} y / d z^{2}+(1 / z) d y / d z+(1-$ $\left.\mu^{2} / z^{2}\right) y=0 \quad$ with $y=\delta w / \sqrt{\rho}, \quad z=\sqrt{\Lambda\left(\Omega^{2}+1 / 4\right)} \bar{\rho}$, and $\mu=i \varpi$, where $\varpi=\sqrt{2 \Omega^{2}+1 / 4}$. Thus, the general solution for $\delta w$ can be written as

$$
\delta w=c_{1} \sqrt{\bar{\rho}} J_{\mu}(z)+c_{2} \sqrt{\bar{\rho}} Y_{\mu}(z),
$$

where $c_{1,2}$ are some constants, and $J_{\mu}(z)$ and $Y_{\mu}(z)$ are the standard Bessel functions of the first and second kinds, respectively. Since $\mu$ is purely imaginary here, instead of $J_{\mu}(z)$ and $Y_{\mu}(z)$, it is better to use the pair of real functions [15]

$$
\begin{aligned}
& F_{\mu}(z)=\frac{1}{2} \sec \left(\frac{\mu \pi}{2}\right)\left\{J_{\mu}(z)+J_{-\mu}(z)\right\}, \\
& G_{\mu}(z)=\frac{1}{2} \csc \left(\frac{\mu \pi}{2}\right)\left\{J_{\mu}(z)-J_{-\mu}(z)\right\} .
\end{aligned}
$$

Their power series representations read

$$
\begin{aligned}
F_{i \varpi}(z)= & \left(\frac{2 \varpi \tanh (\varpi \pi / 2)}{\pi}\right)^{1 / 2} \\
& \times \sum_{n=0}^{\infty} \frac{(-1)^{n}\left(z^{2} / 4\right)^{n} \cos \left(\varpi \ln (z / 2)-\phi_{\varpi, n}\right)}{n !\left[\left(\varpi^{2}\right)\left(1+\varpi^{2}\right) \cdots\left(n^{2}+\varpi^{2}\right)\right]^{1 / 2}},
\end{aligned}
$$

$$
\begin{aligned}
G_{i \varpi}(z)= & \left(\frac{2 \varpi \operatorname{coth}(\varpi \pi / 2)}{\pi}\right)^{1 / 2} \\
& \times \sum_{n=0}^{\infty} \frac{(-1)^{n}\left(z^{2} / 4\right)^{n} \sin \left(\varpi \ln (z / 2)-\phi_{\varpi, n}\right)}{n !\left[\left(\varpi^{2}\right)\left(1+\varpi^{2}\right) \cdots\left(n^{2}+\varpi^{2}\right)\right]^{1 / 2}},
\end{aligned}
$$

where $\phi_{\varpi, n}=\arg \{\Gamma(1+n+i \varpi)\}$.

To obtain the transfer matrix $R$ for propagating the envelope deviations

$$
\left[\begin{array}{c}
\delta w \\
\delta w^{\prime}
\end{array}\right]=R\left[\begin{array}{c}
\delta w(0) \\
\delta w^{\prime}(0)
\end{array}\right]
$$

we note that

$$
\left[\begin{array}{c}
\delta w \\
\delta w^{\prime}
\end{array}\right]=A\left[\begin{array}{c}
y \\
y^{\prime}
\end{array}\right], \quad A=\left[\begin{array}{cc}
\sqrt{\bar{\rho}} & 0 \\
\frac{1}{2 \sqrt{\bar{\rho}}} & \sqrt{\bar{\rho}}
\end{array}\right],
$$


and

$$
\left[\begin{array}{c}
y \\
y^{\prime}
\end{array}\right]=R_{y}\left[\begin{array}{c}
y \\
y^{\prime}
\end{array}\right]_{0}, \quad R=A(\bar{\rho}) R_{y} A\left(\bar{\rho}_{0}\right)^{-1},
$$

where

$$
R_{y}=\left[\begin{array}{ll}
F_{i \varpi} & G_{i \varpi} \\
F_{i \varpi}^{\prime} & G_{i \varpi}^{\prime}
\end{array}\right]\left[\begin{array}{ll}
F_{i \varpi}(0) & G_{i \varpi}(0) \\
F_{i \varpi}^{\prime}(0) & G_{i \varpi}^{\prime}(0)
\end{array}\right]^{-1} .
$$

In computing $R_{y}$, we can ignore any constant coefficients in $F_{i \varpi}$ and $G_{i \varpi}$. It is also sufficient to keep the zero-order term for the initial values. After a little algebra, we have

$$
\begin{aligned}
R_{y}= & \sum_{n=0}^{\infty} \frac{(-1)^{n}\left(z^{2} / 4\right)^{n}}{n !\left[\left(1+\varpi^{2}\right) \cdots\left(n^{2}+\varpi^{2}\right)\right]^{1 / 2}} \\
& \times\left[\begin{array}{cc}
\cos u_{n} & \bar{\rho}_{0} \\
-\frac{\varpi}{\sigma}\left(\sin u_{n}\right. \\
\left.\overline{\bar{\rho}} u_{n}-\frac{2 n}{\varpi} \cos u_{n}\right) & \frac{\bar{\rho}_{0}}{\bar{\rho}}\left(\cos u_{n}+\frac{2 n}{\varpi} \sin u_{n}\right)
\end{array}\right],
\end{aligned}
$$

where $u_{n}=\varpi \ln \left(\bar{\rho} / \bar{\rho}_{0}\right)-\phi_{\varpi, n}+\phi_{\varpi, 0}$. The phase difference $\phi_{\varpi, n}-\phi_{\varpi, 0}$ can be computed as

$$
\begin{aligned}
& \arg \{\Gamma(1+n+i \varpi)\}-\arg \{\Gamma(1+i \varpi)\} \\
& \quad=\arg [\Gamma(1+n+i \varpi) \Gamma(1-i \varpi)] \\
& \quad=\arg [(n+i \varpi) \cdots(1+i \varpi) \Gamma(1+i \varpi) \Gamma(1-i \varpi)] \\
& \quad=\frac{\pi \varpi}{\sinh (\pi \varpi)} \arg [(n+i \varpi)(n-1+i \varpi) \cdots(1+i \varpi)] .
\end{aligned}
$$

Combining Eqs. (A10), (A9), and (A12), we get the transfer matrix

$$
R=\sum_{n=0}^{\infty} \frac{(-1)^{n}\left(z^{2} / 4\right)^{n}}{n !\left[\left(1+\varpi^{2}\right) \cdots\left(n^{2}+\varpi^{2}\right)\right]^{1 / 2}}\left[\begin{array}{ll}
r_{11} & r_{12} \\
r_{21} & r_{22}
\end{array}\right],
$$

where

$$
\begin{aligned}
& r_{11}=\sqrt{\frac{\bar{\rho}}{\bar{\rho}_{0}}}\left(\cos u_{n}-\frac{1}{2 \varpi} \sin u_{n}\right), \\
& r_{12}=\frac{\sqrt{\bar{\rho} \bar{\rho}_{0}}}{\varpi} \sin u_{n}, \\
& r_{21}=-\frac{\varpi}{\sqrt{\bar{\rho} \bar{\rho}_{0}}}\left[\left(1+\frac{1+4 n}{4 \varpi^{2}}\right) \sin u_{n}-\frac{2 n}{\varpi} \cos u_{n}\right], \\
& r_{22}=\sqrt{\frac{\bar{\rho}_{0}}{\bar{\rho}}}\left(\cos u_{n}+\frac{1+4 n}{2 \varpi} \sin u_{n}\right) .
\end{aligned}
$$

Note that at $n=0$ this matrix reduces to the previous result $[3,4,14]$ except for a factor $\gamma^{\prime} \bar{\rho} / \gamma$ different in the offdiagonal elements (because $\delta w^{\prime}$ is a differentiation with respect to $\bar{\rho}$ instead of $s$ ). The $n>0$ terms give the effects due to emittance.

[1] B. E. Carlsten, Nucl. Instrum. Methods Phys. Res., Sect. A 285, 313 (1989).

[2] X. Qiu, K. Batchelor, I. Ben-Zvi, and X.-J. Wang, Phys. Rev. Lett. 76, 3723 (1996).

[3] L. Serafini and J. B. Rosenzweig, Phys. Rev. E 55, 7565 (1997).

[4] C.-x. Wang, Phys. Rev. E 74, 046502 (2006).

[5] C.-x. Wang, K.-J. Kim, M. Ferrario, and A. Wang, Phys. Rev. ST Accel. Beams 10, 104201 (2007).

[6] J.D. Lawson, The Physics of Charged Particle Beams (Oxford University Press, New York, 1988), 2nd ed.

[7] M. Ferrario et al., Phys. Rev. Lett. 99, 234801 (2007).

[8] A. Chanchi et al., Phys. Rev. ST Accel. Beams 11, 032801 (2008).

[9] G. Lambertson, L. J. Laslett, and L. Smith, IEEE Trans. Nucl. Sci. 24, 993 (1977).

[10] L. Serafini and M. Ferrario, SPIE-LASER' 99 Conference, San Jose, CA, 1999, p. 2.

[11] C.-x. Wang, Nucl. Instrum. Methods Phys. Res., Sect. A 557, 94 (2006).

[12] M. Ferrario and L. Serafini, in Proceedings of the 6th European Particle Accelerator Conference, Stockholm, 1998 (IOP, London, 1998), p. 1271.

[13] See, for example, H. Wiedemann, Particle Accelerator Physics (Springer-Verlag, Berlin, 1993).

[14] J. Rosenzweig and L. Serafini, Phys. Rev. E 49, 1599 (1994).

[15] T. M. Dunster, SIAM J. Math. Anal. 21, 995 (1990). 